\title{
Colonic Perforation Secondary to Idiopathic Intramural Hemorrhage
}

\author{
Takashi Sakamoto, Akira Saito ${ }^{1}$, Alan Kawarai Lefor ${ }^{2}$, Tadao Kubota \\ Departments of Surgery and ${ }^{1}$ Pathology, Tokyo Bay Urayasu Ichikawa Medical Center, Urayasu; ${ }^{2}$ Department of Surgery, Jichi Medical \\ University, Shimotsuke, Japan
}

Intramural colonic hemorrhage is rare and often secondary to trauma or anticoagulation therapy. Idiopathic intramural hemorrhages in the alimentary tract have rarely been reported. While several reports of spontaneous perforation of an intramural rectal hematoma have been published, no reports of spontaneous perforation in the ascending colon due to a hematoma have. We describe a patient with an ascending colonic perforation secondary to spontaneous intramural hemorrhage. The patient is a 35-year-old male, who presented with acute abdominal pain and no history of trauma. An abdominal computed tomography scan showed a high-density area around the ascending colon, and nonoperative management was instituted. On the eighth hospital day, the pain worsened, and abdominal computed tomography scan showed free air. An emergent right hemicolectomy was performed. Intramural hematoma and ischemia with perforation, with no obvious etiology, were found. The patient was discharged on the 14th postoperative day.

Keywords: Intramural hemorrhage; Colon; Perforation

\section{INTRODUCTION}

An intramural colonic hemorrhage is rare and when found is usually secondary to blunt trauma or anticoagulation. Several reports have described colonic perforation secondary to intramural hemorrhage in the rectum $[1,2]$. However, to the best of our knowledge, no reports of spontaneous perforation in the ascending colon due to intramural hemorrhage have been published. Furthermore, published reports on idiopathic intramural hemorrhage in the alimentary tract are rare [3]. We present the case of a patient with colonic perforation secondary to an idiopathic intramural hemorrhage.

Received: July 11,2016 - Accepted: November 2, 2016

Correspondence to: Takashi Sakamoto, M.D.

Department of Surgery, Tokyo Bay Urayasu Ichikawa Medical Center,

3-4-32, Todaijima, Urayasu, Chiba, 279-0001, Japan

Tel: +81-47-351-3101, Fax: +81-47-352-6237

E-mail: takac93@gmail.com

(C) 2016 The Korean Society of Coloproctology

This is an open-access article distributed under the terms of the Creative Commons Attribution NonCommercial License (http://creativecommons.org/licenses/by-nc/4.0) which permits unrestricted noncommercial use, distribution, and reproduction in any medium, provided the original work is properly cited.

\section{CASE REPORT}

A 35-year-old male with a history of untreated hypertension presented to an outside facility with acute onset of abdominal pain accompanied by a small amount of hematochezia. He had no history of trauma or anticoagulation therapy. He appeared comfortable and had normal vital signs. Physical examination revealed localized abdominal tenderness in the right upper quadrant. Laboratory studies were within normal limits except for a white blood cell count of $19,200 / \mu \mathrm{L}$ and a serum creatinine of $1.27 \mathrm{mg} / \mathrm{dL}$. Serum hemoglobin was $14.7 \mathrm{~g} / \mathrm{dL}$ and coagulation studies showed a prothrombin time normalized international ratio and an activated partial thromboplastin time of 0.94 and 23.6, respectively. An abdominal computed tomography scan (Fig. 1A) showed an area of increased density in the hepatic flexure and ascending colon. At this point, diagnostic considerations included ischemic colitis and diverticulitis.

Based on the patient's overall situation, the diagnosis of diverticulitis was made. The patient was admitted, and nonoperative management, including no oral intake and administration of cefazolin, was begun. On the fifth hospital day, the abdominal pain persisted, and laboratory tests showed anemia (hemoglobin decreased from $14.7 \mathrm{~g} / \mathrm{dL}$ to $7.0 \mathrm{~g} / \mathrm{dL}$ ). A contrast-enhanced com- 


\begin{aligned} & Annals of $\begin{array}{l}\text { Colonic Perforation Secondary to Idiopathic Intramural Hemorrhage } \\ \text { Takashi Sakamoto, et al. }\end{array} \\ &$\hline\end{aligned}
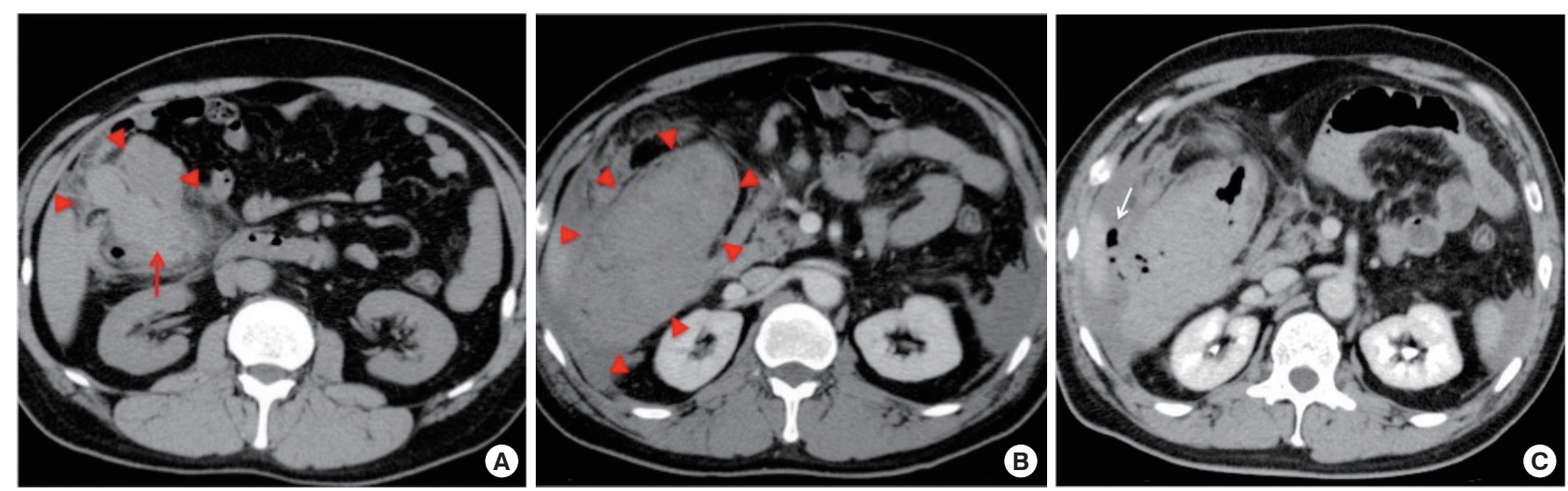

Fig. 1. Computed tomography scans of the abdomen. (A) High-density area consistent with a hematoma or inflammation is seen around the ascending colon on hospital day 1 (red arrowheads). Red arrow means colonic lumen. (B) An enlarged hematoma is seen (red arrowheads) without free air or ascites. (C) Ascites is present anterior to the liver with free air (white arrow) lateral to the ascending colon.

puted tomography scan was performed, which showed enlargement of the high-density area (Fig. 1B). The patient was then transferred to our hospital with the diagnosis of a large hematoma around the ascending colon. At the time of transfer, vital signs included a temperature of $38.3^{\circ} \mathrm{C}$, a blood pressure of $179 / 128$ $\mathrm{mmHg}$, a pulse of 103 beats/min, and a respiratory rate of 16 breaths/min. Physical examination showed mild tenderness in the right upper quadrant. Nonoperative management was continued, with no oral intake and no antibiotic administration. Because the systolic blood pressure had been greater than $170 \mathrm{mmHg}$, nifedipine was given to limit additional bleeding due to high blood pressure. On the eighth hospital day, although the white blood cell count remained elevated at $12,700 / \mu \mathrm{L}$, the serum hemoglobin was increased to $7.7 \mathrm{~g} / \mathrm{dL}$, and abdominal symptoms and physical findings were resolving, so oral intake was started.

Six hours after eating, the patient complained of recurrent and more severe abdominal pain. Physical exam revealed significant abdominal tenderness in the right upper quadrant. A computed tomography scan (Fig. 1C) showed free air in the abdominal cavity, so an emergent laparotomy was performed with a presumptive diagnosis of colonic perforation. Intraoperatively, a massive hematoma was found posterior to the ascending colon. The odor of feces was present, but no spillage of stool was noted. A right hemi-colectomy was performed.

Intraoperative examination of the specimen showed an intramural hematoma with an ischemic mucosa. At the center of the area of ischemia, a pinhole perforation was seen (Fig. 2). Histological findings showed hemorrhage spreading from the submucosal layer to the subserosal layer. In the subserosal layer, neutrophils, monocytes, proliferations of capillary vessels, and proliferations of fibroblast cells were seen, suggesting an inflammatory granulation response. These findings support the hypothesis that the subserosal findings were older than the perforation. The hemorrhage preceded the ischemia and perforation (Fig. 3). Based on

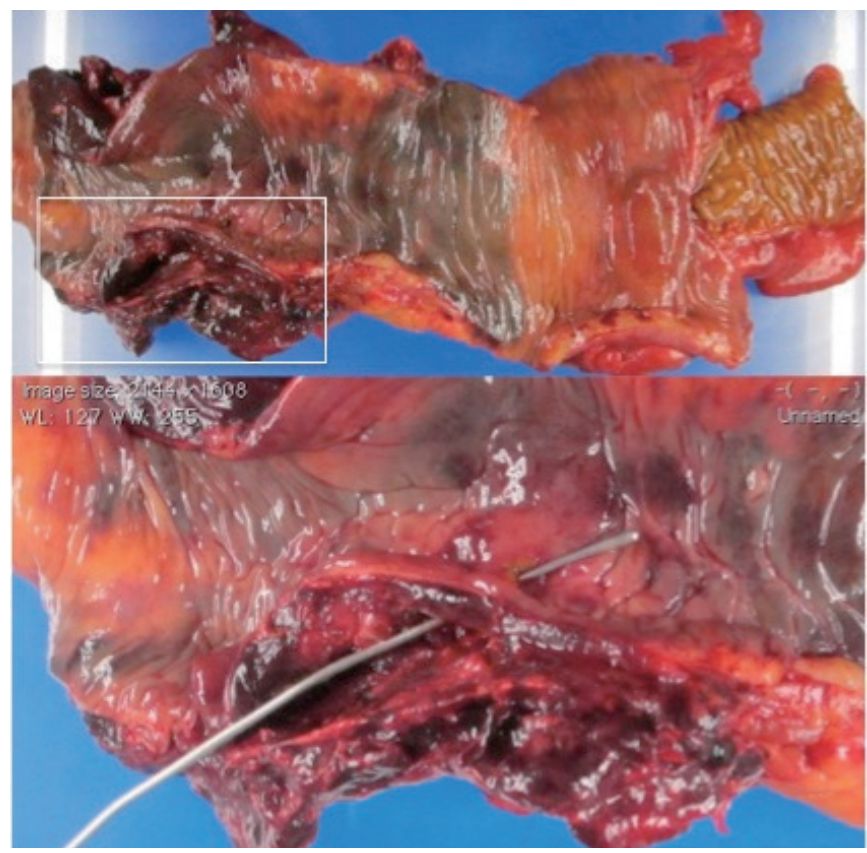

Fig. 2. Intraoperative findings. A large hematoma was found deep to the subserosa with an apparent demarcation line of ischemia. A $1-\mathrm{mm}$ perforation was detected in the area of the ischemia and hematoma.

this concept, we believe that spontaneous intramural bleeding resulted in ischemia followed by perforation. The patient was discharged 14 days postoperatively after an uneventful postoperative course.

\section{DISCUSSION}

Intramural hematomas in the alimentary tract are rare. When 

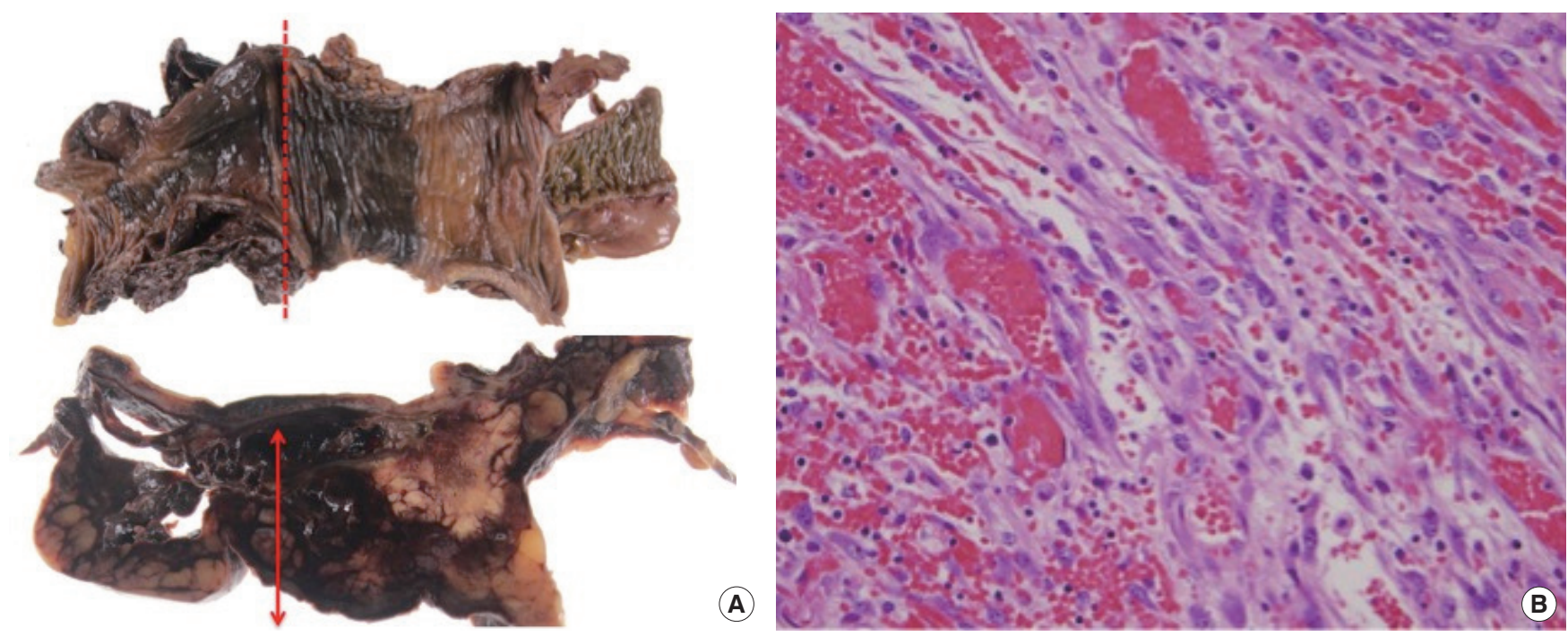

Fig. 3. Pathologic findings. (A) In the gross findings, a hematoma is present from the submucosal to the subserosal layers (red arrow), with partial thickness necrosis and perforation. (B) The subserosa showed inflammation, neutrophils, monocytes, proliferation of capillary vessels and proliferation of fibroblast cells, which indicate an inflammatory granulation response $(\mathrm{H} \& \mathrm{E}, \times 400)$.

they do occur, blunt trauma and anticoagulants are the typical etiologies. The majority of intramural hematomas have been reported in the duodenum and jejunum. Thirty-six percent of these patients were receiving anticoagulant therapy, and $40 \%$ were secondary to trauma [3]. Many patients taking anticoagulant medications have prolonged prothrombin times above the therapeutic range [3]. Colonic intramural hematomas are extremely rare, accounting for less than $5 \%$ of all intramural hematomas [4]. Most patients with intramural hematomas present with intestinal obstruction. Nonoperative therapy is usually sufficient because the hematomas will resorb [2].

Idiopathic colonic intramural hematomas are particularly rare [3]. One report has been published on a patient with a transverse colonic intramural hematoma that was managed nonoperatively; the patient was undergoing antiplatelet therapy [5]. An idiopathic hematoma in the transverse colon without a history of trauma or anticoagulants that was managed nonoperatively was reported by Umeda et al. [4], and an idiopathic hematoma in the transverse colon that was managed surgically was reported by Nozu [6]. However, to the best of our knowledge, no reports of idiopathic intramural hematomas of the colon with perforation have been published. The present patient did not have a history of trauma or hematologic disorders and was not receiving anticoagulant therapy. Based on clinical findings, the perforation occurred 8 days after the hematoma.

The normal mammalian response to injury occurs in 3 stages: inflammation, new tissue formation, and remodeling. The first stage occurs immediately after tissue damage. Neutrophils are recruited to the wound, and in the first few days, monocytes appear in the wound. The second stage of wound healing occurs from 2 to 10 days after injury and is characterized by cellular proliferation and migration of various cells. New blood vessels form, and the sprouts of capillaries associated with fibroblasts and macrophages (monocytes) replace the fibrin matrix with granulation tissue [7]. In this patient, the subserosal layer contained neutrophils, monocytes, proliferations of capillaries, and proliferations of fibroblasts, suggesting an inflammatory granulation response. These histological findings suggest that the hemorrhage preceded ischemia and perforation by more than 2 days (Fig. 3). We believe that spontaneous intramural bleeding led to ischemia, followed by perforation.

The findings in this patient provide indirect evidence of a colonic perforation secondary to an idiopathic intramural hematoma. Another possible explanation is a minor perforation in the area of diverticulitis with bleeding that sealed and then reperforated due to ischemia caused by the hematoma. The imaging studies and pathologic evaluation of the specimen did not show evidence of diverticular disease suggesting an idiopathic etiology, but this can also explain the clinical course of this patient.

In conclusion, colonic perforation secondary to an idiopathic intramural hematoma is very rare. In our patient, enlargement of the hematoma likely led to ischemia and perforation. Thus, a patient with an intramural hematoma in the gastrointestinal tract should be monitored closely, and surgical treatment should be considered if the hematoma is progressively enlarging.

\section{CONFLICT OF INTEREST}

No potential conflict of interest relevant to this article was reported. 


\section{REFERENCES}

1. TerKonda SP, Nichols FC 3rd, Sarr MG. Spontaneous perforating hematoma of the rectum. Report of a case. Dis Colon Rectum 1992;35:270-2.

2. Li ZL, Wang ZJ, Han JG. Spontaneous perforation of an intramural rectal hematoma: report of a case. World J Gastroenterol 2012;18:2438-40.

3. Hughes CE 3rd, Conn J Jr, Sherman JO. Intramural hematoma of the gastrointestinal tract. Am J Surg 1977;133:276-9.
4. Umeda I, Ohta H, Doi T, Nobuoka A, Kanisawa Y, Kawasaki R, et al. Idiopathic intramural hematoma of the colon. Gastrointest Endosc 2007;66:861-4.

5. Liu Y, Yang S, Tong Q. Spontaneous intramural hematoma of colon. Clin Gastroenterol Hepatol 2012;10:e38.

6. Nozu T. Idiopathic spontaneous intramural hematoma of the colon: a case report and review of the literature. Clin J Gastroenterol 2009;2:161-5.

7. Reinke JM, Sorg H. Wound repair and regeneration. Eur Surg Res 2012;49:35-43. 